\title{
A giant right atrial appendage aneurysm in an infant: a case report
}

\author{
Jianming Yue ${ }^{1}$, Qi Wang ${ }^{1}$, Bin Liu ${ }^{1}$, and Leng Zhou ${ }^{1}$ \\ ${ }^{1}$ Sichuan University West China Hospital
}

January 22, 2022

\begin{abstract}
Right atrial appendage aneurysms (RAAAs) are extremely rare in cardiac anomaly. According to the literature, no more than 25 cases have been reported so far, among which only 3 cases were children. Here, we reported an infant with a giant RAAA and severe symptoms. The RAAA was diagnosed by echocardiography and surgically resected under cardiopulmonary bypass. The role of transesophageal echocardiography was very important during the aneurysm resection surgery, which helped surgeons to plan surgical procedures during the surgery and evaluate the surgical effect postoperatively.
\end{abstract}

\section{Title page}

Title

A giant right atrial appendage aneurysm in an infant: a case report

Running title

Right atrial appendage aneurysm

Author

Jianming Yue $\mathrm{MD}^{1,2} \mid$ Qi Wang $\mathrm{MD}^{1,2} \mid$ Bin Liu $\mathrm{MD}^{1,2} \mid$ Leng Zhou $\mathrm{PhD}^{1,2}$

Department of Anesthesiology, West China Hospital, Sichuan university

The Research Units of West China(2018RU012)-Chinese Academy of Medical Sciences, West China Hospital,Sichuan UniversityCorresponding authorLeng Zhou, No. 37 Guoxue Lane, Chengdu 610041, Sichuan Province, China, zhoulenghx@foxmail.com, +86 18980601585AcknowledgementWe thank the patient's guardian for allowing us to publish the case report and to use the images taken.

\section{Supported by none.}

\section{Abstract}

Right atrial appendage aneurysms (RAAAs) are extremely rare in cardiac anomaly. According to the literature, no more than 25 cases have been reported so far, among which only 3 cases were children. Here, we reported an infant with a giant RAAA and severe symptoms. The RAAA was diagnosed by echocardiography and surgically resected under cardiopulmonary bypass. The role of transesophageal echocardiography was very important during the aneurysm resection surgery, which helped surgeons to plan surgical procedures during the surgery and evaluate the surgical effect postoperatively.

\section{KEYWORDS}

right atrial appendage aneurysms, infant, echocardiography, giant

\section{1 | INTRODUCTION}


Atrial aneurysm is rare in cardiac anomaly, most of which are left atrial aneurysms in adults. Right atrial appendage aneurysms (RAAAs) are extremely rare compared to other cardiac aneurysms, especially for infants and pediatric population. According to the literature, no more than 25 cases have been reported so far, among which only 3 cases are infants or neonates. ${ }^{1,2}$ A systematic review published in 2014 found that the highest morbidity of RAAAs was in the third decade of life, with cases noted from the perinatal period to 72 years of age. ${ }^{3}$ RAAAs usually involve free walls and atrial appendages, most have congenital origins and develop in the course of pregnancy. ${ }^{2,4}$ Generally, patients with RAAA are usually asymptomatic and are diagnosed incidentally or until they present some clinical symptoms like arrhythmia or dyspnea. ${ }^{5}$ Pulmonary thromboembolism is a common complication due to blood stasis in the aneurysm. ${ }^{6}$ However, there is no guideline for the treatment of this condition, and surgical resection is safe and can be performed through sternotomy with or without cardiopulmonary bypass for symptomatic patients. ${ }^{2,5}$ Here, we reported an infant with a giant RAAA and severe symptoms who underwent surgical resection of the aneurysm.

\section{2 | CASE REPORT}

A 1-year-old infant was first disclosed right atrial enlargement at the 26 weeks of gestation, but the parents were unable to provide detailed prenatal examination data. The infant had been asymptomatic and had not received any echocardiography until he got a prolonged unhealed cold and eventually developed pneumonia accompanying with supraventricular tachycardia and dyspnea at the local hospital. After the infection and arrhythmia were controlled by antibiotics and digoxin, he was transferred to our hospital.

Preoperative chest X-ray showed an enlarged cardiac shadow with a prominent right atrial contour (Figure 1A). Transthoracic echocardiography (TTE) disclosed a giant right atrial aneurysmal structure measured $7.8 \times 7.5 \mathrm{~cm}$ and a large patent foramen ovale $(\mathrm{PFO})$ (Figure 1B). The anatomical position and structure of the tricuspid valve (TV) were normal. The right ventricle (RV) and left atrium (LA) were compressed by the abnormal cavity, whereas tricuspid regurgitation was minimal. The other valves and left ventricle were normal with good systolic function. An enhanced cardiac computed tomography scan was requested and showed a large aneurysmal dilatation of the right atrial appendage with no internal thrombi (Figure 2 ). Thus, the infant was diagnosed with a giant right atrial appendage aneurysm complicated with a PFO and was indicated to surgery considering the large size of RAAA, the PFO and the previous episodes of supraventricular tachycardia and dyspnea. After induction of anesthesia, transesophageal echocardiography (TEE) was performed to evaluate the RAAA (Figure 3A) and cardiac structure. It revealed that the free wall of the right ventricle (RV) was compressed by the large aneurysm (Figure 3B). Although blood flow in the right atrium and RAAA was slow, no thrombus was found. Both ventricles and left atrium were of normal size and function (Figure 3B). TEE also revealed a large PFO and mild tricuspid regurgitation with normal RV systolic pressure. Considering the large aneurysm might have higher risks of bleeding and arrhythmia, the surgeons performed the aneurysm resection under cardiopulmonary bypass (CPB). The giant RAAA occupied almost all pericardial space (Figure 4A). The aneurysm sac was opened, an extremely thin atrial wall was observed, no thrombus was found and there were many tube-like structures distributed on the inner wall during surgery (Figure 4B). The aneurysm was resected and the PFO was closed. After weaning of CPB, postoperative TEE showed that the cardiac structure was normal and the tricuspid valve functioned normally without regurgitation or insufficiency (Figure 5A). And the postoperative TTE dimension of the right atrial appendage showed a decrease in right atrial size to $2.0 \mathrm{~cm} \times 2.35 \mathrm{~cm}$ (Figure 5B). Later pathological examination confirmed the diagnosis of RAAA (Figure 5C).

The postoperative course was uneventful and the infant was discharged on the 5th day after the surgery. He had a full recovery without any complications at the three-month follow-up.

\section{3 | DISCUSSION}

Compared with left atrial appendage aneurysm, RAAA is a rare cardiac anomaly that was first reported in 2001 which thought to be caused by dysplasia of the muscular wall of the right atrium (RA) and right atrial appendage. ${ }^{7,8}$ Most of these aneurysms affect adults in their third decade of life but they can also occur in children and neonates and can also be detected prenatally. ${ }^{2,3}$ 
The diagnosis can be confirmed by imaging modalities, especially initial echocardiography and chest X-ray. ${ }^{9}$ Both TTE and TEE are very effective in diagnosis and distinguish of the aneurysm, such as pericardial cysts, Ebstein's anomaly, cortriatriatum and thrombosis. ${ }^{10}$ Agitated saline study with echocardiography can be performed to confirm the diagnosis and to distinguish from pericardial effusion or cysts, demonstrating opacification of the RAAA and RV. ${ }^{2}$ TEE plays an important role in further preoperative evaluation and planning surgical procedures during resection of RAAA. In this case, before the aneurysm resection TEE presented the giant RAAA, PFO and RV compression. During the surgery, surgeons concerned the resection of the aneurysm might change the entire anatomical structure, leading to tricuspid valve dysfunction. Additionally TEE played a key role in confirming valve competency during the surgery. In this case, the postoperative TEE confirmed that the tricuspid valve still functioned normally without regurgitation or insufficiency. Therefore, the patient was not indicated to tricuspid valvuloplasty. Taken together, TEE is very helpful for surgeons to formulate detailed surgical plan and evaluate the effect of surgical correction during cardiac surgery. Finally, TTE is useful for long-term follow-up on this case since there is no report regarding postoperative recurrence or death after RAAA resection.

Although RAAA is a very rare anomaly in pediatric patients, prenatal diagnosis via fetal echocardiography is possible. Clinical experience of treatment for RAAA is limited and the natural history and optimal management strategy are still unclear. Therefore, the therapeutic strategies of RAAA remain controversial and aneurysm resection and anticoagulation therapy are not recommended in all cases. ${ }^{3}$ Surgical resection is preferred for patients with symptoms or progressive enlargement in preventing thromboembolisms and lowering the risk of atrial arrhythmia and heart failure, which are the most common complications of these aneurysms. ${ }^{2,3}$ Oral anticoagulants is recommended to reduce the risk of thromboembolic events in patients with contraindications to surgery. ${ }^{4}$

In conclusion, echocardiography plays an important role in the diagnosis and surgical resection of RAAA as well as postoperative evaluation of surgical effect. Treatment should be modified according to the age, presentation, imaging findings and the follow-up results.

\section{CONFLICTS OF INTEREST}

The authors have no conflicts of interest to disclose.

\section{INFORMED CONSENT}

Written informed consent was obtained from the patient's guardian for the publication of this case report and the accompanying images.

\section{DATA AVAILABILITY STATEMENT}

No additional data.

\section{ORCID}

Jian-Ming Yue: 0000-0002-1826-4931

Qi Wang: 0000-0003-0509-3851

Biu Liu: 0000-0001-5503-0053

Leng Zhou: 0000-0003-3374-7007

\section{Author contributions:}

All the authors participated in the management of this patient and provided suggestion for the manuscript. Jian-Ming Yue followed up the patient closely, collected the data and written the manuscript. Qi Wang and Bin Liu provided some suggestions about the manuscript and management of this patient. Leng zhou reviewed the manuscript and was the corresponding author.

\section{References}


1. Bachani N, Jadwani J, Joshi S, et al. Right atrial appendage aneurysm with intractable atrial tachycardia. IHJ Cardiovascular Case Reports (CVCR). 2019;3(3):108-111.

2. Tunks RD, Malysz J, Clark JB. Neonatal Management of a Giant Right Atrial Appendage Aneurysm. Circulation. 2015;132:e226-228.

3. Aryal MR, Hakim FA, Giri S, et al. Right atrial appendage aneurysm: a systematic review. Echocardiography. 2014;31:534-539.

4. Barberato SH, Barberato MF, Avila BM, et al. Aneurysm of the right atrial appendage. Arq Bras Cardiol. 2002;78:236-241.

5. Sivakumaran L, Sayegh K, Mehanna E, et al. Use of cardiovascular magnetic resonance in the evaluation of a giant right atrial appendage aneurysm: a case report and review of the literature. BMC Res Notes. 2017;10:681.

6. Toufan M, Pourafkari L, Akbarzadeh F, et al. A case of right atrial appendage aneurysm mimicking a pericardial cyst on echocardiogram. Echo Res Pract. 2014;1:K5-8.

7. Ishii Y, Inamura N, Kayatani F. Congenital aneurysm of the right atrial appendage in a fetus. Pediatr Cardiol. 2012;33:1227-1229.

8. Mizui S, Mori K, Kuroda Y. Ectopic atrial tachycardia due to aneurysm of the right atrial appendage. Cardiol Young. 2001;11:229-232.

9. Moraes F, Santos CL, Lira V, et al. Congenital aneurysm of the right atrium. Eur J Cardiothorac Surg. 2001;19:943-944.

10. Pontecorboli G, Figueras I Ventura RM, Carlosena A, et al. Use of delayed-enhancement magnetic resonance imaging for fibrosis detection in the atria: a review. Europace. 2017;19:180-189.

\section{Figure legends}

FIGURE 1 Chest X-ray showed cardiomegaly with enlarged right atrial contour (A); The subxiphoid view of TTE showed the giant RAAA and the PFO (B); The apical four-chamber view showed large aneurysm, measuring $7.8 \times 7.5 \mathrm{~cm}(\mathrm{C})$. a = aneurysm; LA: left atrium; LV: left ventricle; MV: mitral valve; PFO = patent foramen ovale; RA: right atrium; RAAA = right atrial appendage aneurysm; RV: right ventricle; $\mathrm{TTE}=$ transthoracic echocardiography; TV: tricuspid valve.

FIGURE 2 Enhanced cardiac computed tomography showed a large dilatation of the right atrium and atrial appendage. LA: left atrium; LV: left ventricle; RA: right atrium; RAAA = right atrial appendage aneurysm; RV: right ventricle.

FIGURE 3 The intraoperative TEE showed the thin wall of right atrial appendage aneurysm with a size of $6.1 \times 7.9 \mathrm{~cm}(\mathrm{~A})$; The 4-chamber view showed the right ventricle was compressed by the atrial appendage aneurysm (B). $\mathrm{LA}=$ left atrium; $\mathrm{LV}=$ left ventricle; $\mathrm{MV}=$ mitral valve; $\mathrm{RA}=$ right atrium; $\mathrm{RAAA}=$ right atrial appendage aneurysm; $\mathrm{RV}=$ right ventricle; $\mathrm{TV}=$ tricuspid valve.

FIGURE 4 The giant RAAA was found upon opening the pericardium (A); The resected sac of right atrial appendage aneurysm (B). RAAA = right atrial appendage aneurysm.

FIGURE 5 The postoperative TEE showed a normal cardiac structure and valve function (A); The postoperative TTE showed a decreased size of right atrium (B); Histopathological sections of the aneurysm showed thinned myocardium and proliferated blood vessels. (x100) (C). LA = left atrium; LV = left ventricle; MV $=$ mitral valve; $\mathrm{RA}=$ right atrium; $\mathrm{RV}=$ right ventricle; $\mathrm{TV}=$ tricuspid valve. 

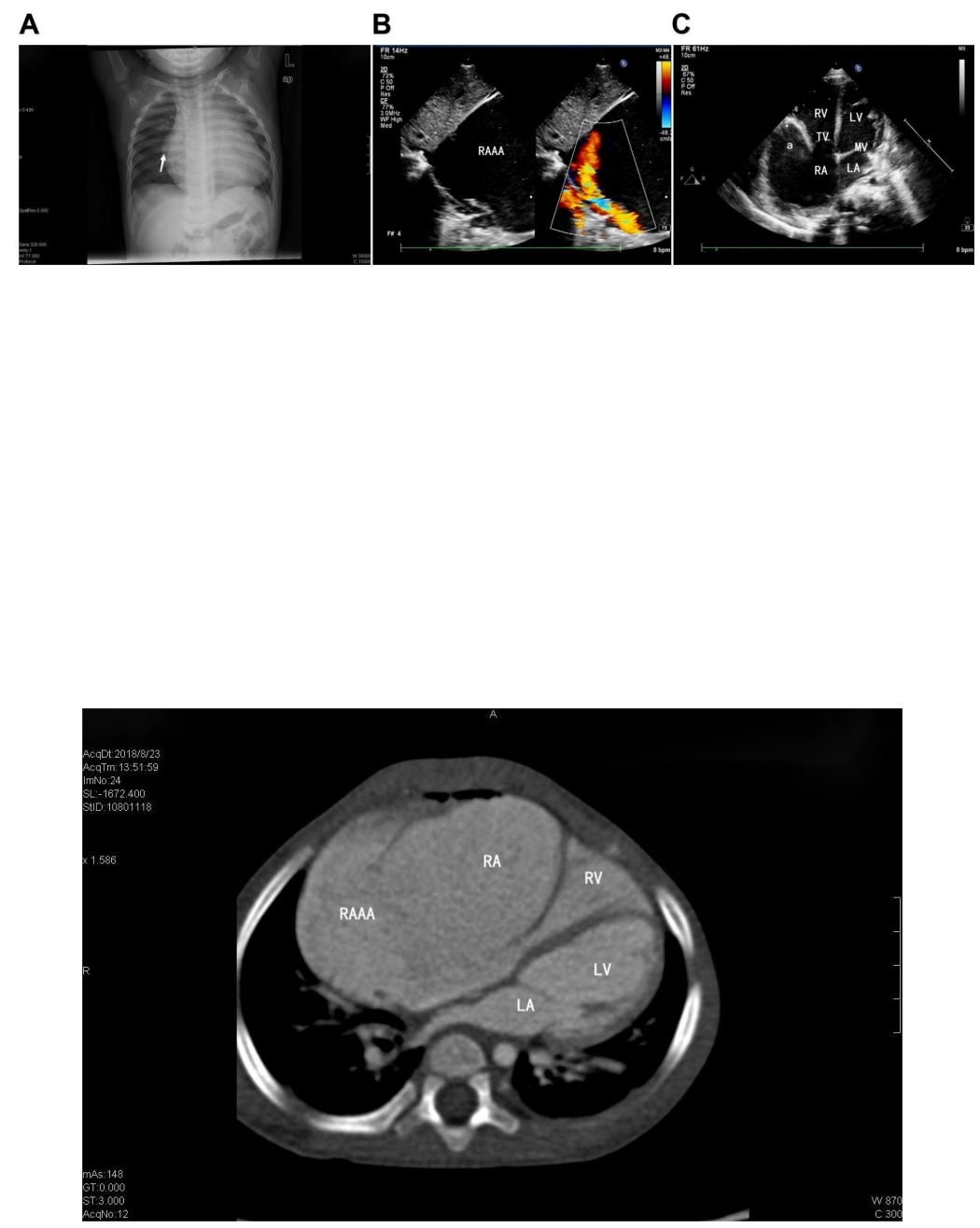

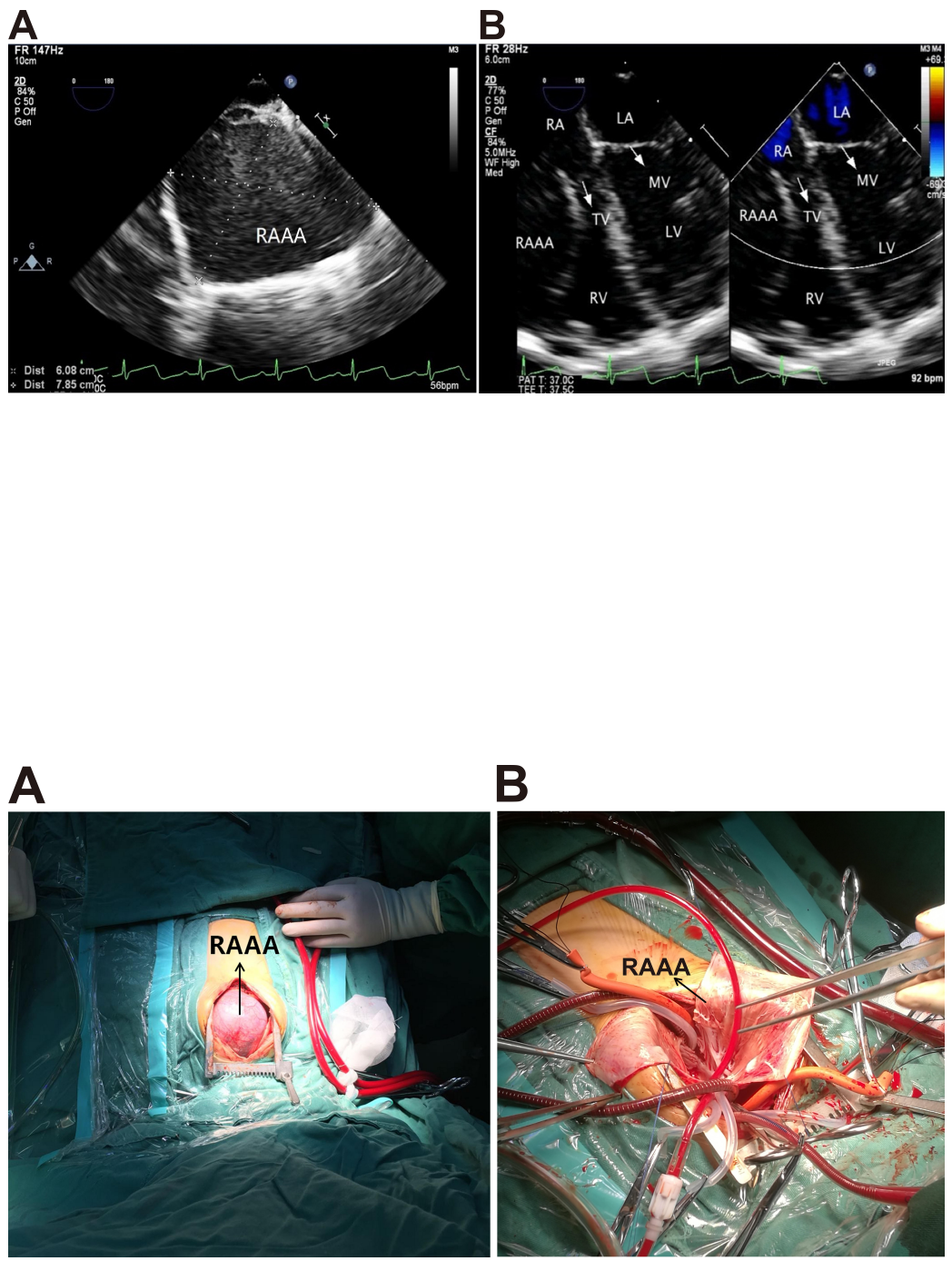

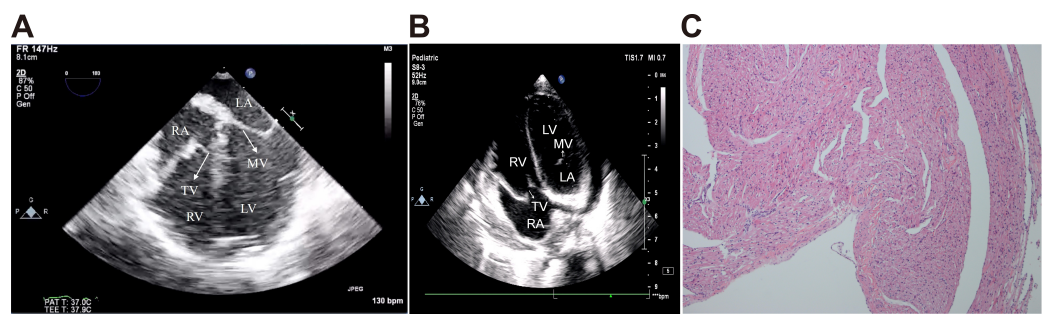